\title{
Accuracy of robot-assisted pedicle screw insertion in adolescent idiopathic scoliosis: is triggered electromyographic pedicle screw stimulation necessary?
}

\author{
K. Aaron Shaw ${ }^{1}$, Joshua S. Murphy², Dennis P. Devito \\ ${ }^{1}$ Department of Orthopaedic Surgery, Dwight D. Eisenhower Army Medical Center, Fort Gordon, GA, USA; ${ }^{2}$ Department of Pediatric \\ Orthopaedics, Children's Healthcare of Atlanta Scottish Rite, Atlanta, GA, USA \\ Contributions: (I) Conception and design: JS Murphy, DP Devito; (II) Administrative support: None; (III) Provision of study materials or patients: DP \\ Devito; (IV) Collection and assembly of data: KA Shaw; (V) Data analysis and interpretation: JS Murphy, DP Devito; (VI) Manuscript writing: All \\ authors; (VII) Final approval of manuscript: All authors. \\ Correspondence to: K. Aaron Shaw, DO. Department of Orthopaedic Surgery, 300 East Hospital Road, Fort Gordon, GA 30905, USA. \\ Email: kenneth.a.shaw34.mil@mail.mil.
}

Background: Screw malpositioning is an identifiable cause of intraoperative neurophysiologic changes. Although triggered screw electromyography (t-EMG) has been found to exhibit high sensitivity for identifying malpositioned screws, no previous study has assessed the utility of combining t-EMG with robotic-assisted pedicle screw placement for identifying malpositioned screws. We sought to evaluate the utility of t-EMG used in combination with robotic-assisted pedicle screw placement for identifying malpositioned screws in patients with adolescent idiopathic scoliosis (AIS).

Methods: Patients undergoing robotic-assisted posterior spinal fusion with pedicle screw fixation for AIS underwent retrospective review from a single surgeons prospectively collected database. Preoperative demographic data and curve characteristics were recorded. Computed tomography (CT) scans were reviewed, measuring pedicle width and classifying pedicle morphology using the channel classification system. Pedicle data was compared against intra-operative t-EMG data, with a minimal threshold of $8 \mathrm{~mA}$ used for screw removal and screw path examination and the rate of screw re-direction recorded. All pedicle screws were verified using image intensification.

Results: Forty-nine patients (11 males, 38 females, average age 14.49 years) with an average curve magnitude of 51 degrees and placement of 844 pedicle screws to attain an average curve correction of $67.7 \%$. The incidence of an absent pedicle (type C or D morphology) was $2 \%$. Overall, 24 screws $(2.8 \%)$ were identified with an abnormal t-EMG threshold. All screws were found to have an intact medial wall upon probing and were reinserted without re-direction. No patient or curve characteristic was predictive of abnormal t-EMG amplitude but smaller pedicles correlated with smaller amplitudes.

Conclusions: With precise pre-operative planning, robotic-assisted pedicle screw placement has shown to be a safe and effective method in treating AIS patients as shown by the lack of medial pedicle breach and malpositioned screws. We found no evidence to support combined use of t-EMG for identifying medially malpositioned screws.

Keywords: Adolescent idiopathic scoliosis (AIS); robotic-assistance; pedicle screw fixation; triggered screw electromyography (t-EMG); neuromonitoring

Submitted Sep 07, 2017. Accepted for publication Apr 04, 2018.

doi: 10.21037 /jss.2018.04.01

View this article at: http://dx.doi.org/10.21037/jss.2018.04.01 


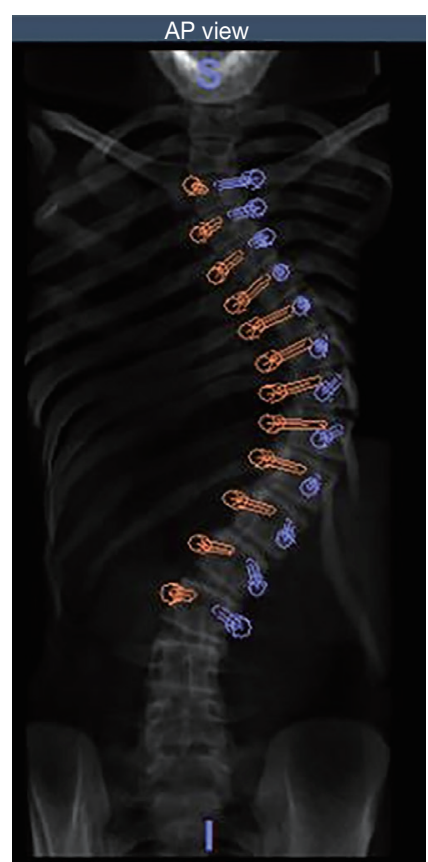

Figure 1 Clinical image of instrumentation planning performed with Renaissance ${ }^{\circledR}$ planning software.

\section{Introduction}

Neurologic complications of spine deformity surgery in pediatric patients are rare, but potentially devastating (1-3). Numerous strategies have been developed and implemented to help reduce the incidence of these complications by ensuring adequate pedicle screw placement and providing early recognition of changes in neurophysiology (4-8). Neuromonitoring has become the standard of care with regard to monitoring for changes in neurophysiology, with numerous modalities by which to monitor the sensory and motor pathways of the nervous system $(1,4,7,9)$. These modalities have excellent sensitivity and specificity for identifying neurologic compromise allowing for early intervention to prevent permanent neurologic changes $(3,10)$.

Pedicle screw malposition is another means by which neurologic compromise can be precipitated, with an incidence reported to be as high as $40 \%$ with an estimated $1 \%$ rate of neurologic complication (11). Triggered screw electromyography (t-EMG) has been used to help identify malpositioned screws, with threshold stimulation varying based upon the spinal segment tested (lumbar versus thoracic), location within the curve (apical versus nonapical) and laterality with regard to the curve (concavity versus convexity) $(3,10,12)$.
Screw navigation is another means by which to minimize malpositioned screws. Several different modalities have been introduced based upon preoperative or intra-operative fluoroscopy or computed tomography (CT), reporting high accuracy for screw placement $(6,8,11,13)$. Kassis et al. (11) reported on the combination of t-EMG with an O-arm based navigation system, finding that t-EMG helped identify malpositioned screws, minimizing the need for a second $\mathrm{O}$-arm scan, minimizing the patient's radiation exposure. Robotic-assisted pedicle screw placement is another navigation modality, combining both preoperative CT scans with intraoperative fluoroscopic imaging to provide excellent accuracy for safe pedicle screw placement $(8,14,15)$. The purpose of this study is to report on the clinical utility of combining t-EMG with robotic-assisted screw placement in patients that underwent a posterior spinal fusion for adolescent idiopathic scoliosis (AIS).

\section{Methods}

\section{Study design and population}

After obtaining approval from our Institutional review board, consecutive patients undergoing posterior spinal fusion for AIS with robotic-assisted pedicle screw placement using the Renaissance ${ }^{\circledR}$ (Mazor Robotics, Caesarea, Israel) were identified for retrospective review. Patients were excluded if they were $\geq 20$ years old at the time of surgery, their procedure was performed for alternative diagnoses, to include Scheuermann's kyphosis, neuromuscular scoliosis, early onset scoliosis, congenital scoliosis, or if they had undergone a previous spinal surgery. All surgeries were performed by a single, high volume pediatric orthopaedic spine surgeon ( $>100$ posterior spinal fusions/year) with 10 years' experience with robotic-assisted pedicle screw insertion and 30 years of clinical experience in the surgical treatment of pediatric spinal conditions. Demographic data was recorded, including patient age and gender.

\section{Surgery}

Patients underwent a low dose 0.5 to $2 \mathrm{~mm} 64$ slice spiral CT scan, performed in the supine position prior to surgery. Each CT scan was analyzed and pedicle screw placement was planned using the Renaissance ${ }^{\circledR}$ planning software (Figure 1). Planning included pedicle screw insertion angle, trajectory, position, diameter, and length. All patients underwent a posterior midline approach to the appropriate 


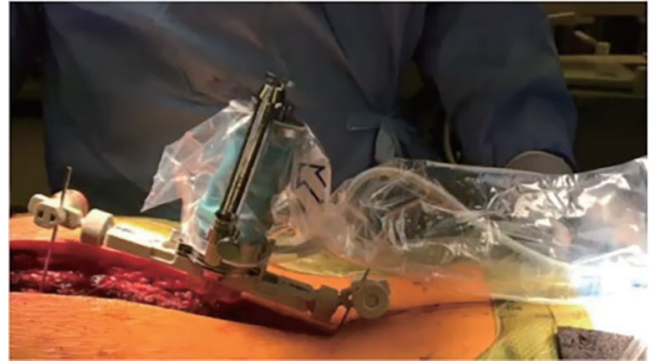

Figure 2 Intraoperative image depicting patient in prone position following posterior midline approach with Renaissance ${ }^{\circledR}$ platform mounted to the posterior spinous processes using Kirschner wire fixation and the robot was attached to the mounting frame.
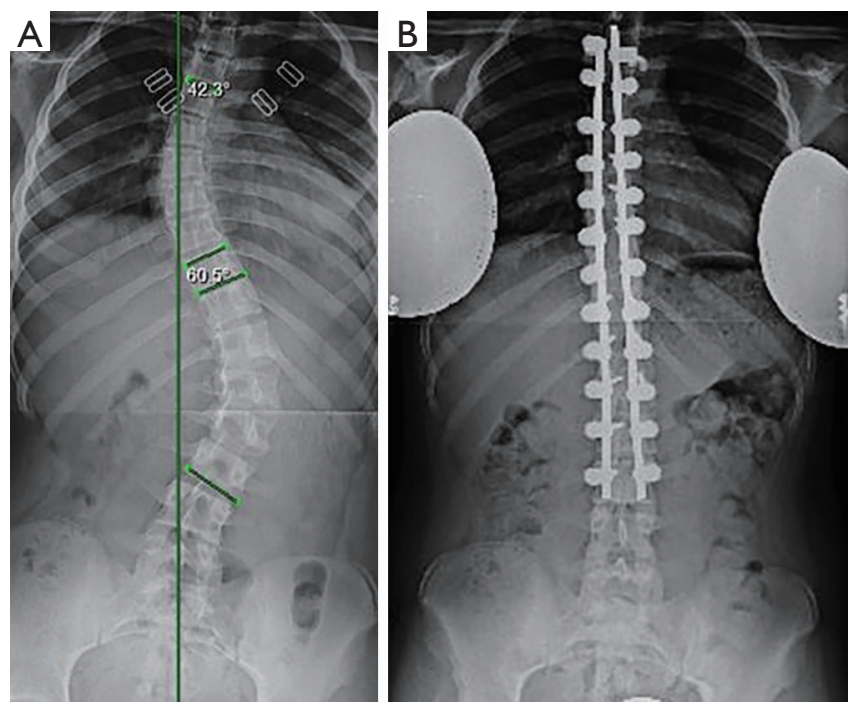

Figure 3 Posteroanterior image of a 12.8-year-old female with a 60-degree Lenke 6C curvature before (A) and following (B) posterior spinal fusion.

spinal levels in the prone position. The Renaissance ${ }^{\circledR}$ Guidance system was attached at specified vertebral level and intraoperative fluoroscopic imaging was performed in the anteroposterior and oblique planes allowing for registration with the preoperative CT images. Following registration, the Renaissance ${ }^{\circledR}$ platform was mounted to the posterior spinous processes using Kirschner wire fixation and the robot was attached to the mounting frame (Figure 2). Pedicle screw tracks were drilled using a $2-0 \mathrm{~mm}$ drill through a robot-guided drill sleeve. Pedicle screw tracks were drilled sequentially prior to performing facetectomies or posterior spinal osteotomies. Using the robot-assisted drill holes, pedicle screws were inserted. All patients were treated with the same top-loading, polyaxial pedicle fixation system (PASS LP, Medicrea International, Neyron France) and included transverse process and pedicle hooks at the upper instrumented vertebral levels. Surgical correction was obtained primarily using the simultaneous translation on 2 rods technique. All pedicle screws underwent triggeredelectromyography stimulation prior to rod insertion and derotation (Figure 3).

\section{Neuromonitoring and pedicle screw stimulation}

All patients received somatosensory-evoked potential (SSEP) and transcranial MEP monitoring during the surgical procedure. Pedicle screw stimulation was performed using DS7A current stimulation (Digitimer North America, LLC; Fort Lauderdale, FL) to generate and deliver square wave constant-current pulses to each pedicle screw following insertion. We used repetitive 4-pulse trains, applied with an interpulse interval of $2 \mathrm{msec}$ between trains and an intertrain rate of $3 \mathrm{~Hz}$. Maximal stimulus delivered varied between cases with maximums of 20,30 , or $40 \mathrm{~mA}$. Minimum stimulus intensities were recorded for each level with a pedicle screw. All screws demonstrating a minimum intensity $\geq 30 \mathrm{~mA}$ was defined as a maximal intensity. All thresholds $\leq 8 \mathrm{~mA}$ were defined as abnormal and underwent screw removal and probing of the pedicle track using a balltip probe. Any track without a palpable medial wall was defined as aberrant and underwent screw re-direction.

\section{Pedicle morphology}

Preoperative radiographs were analyzed with fulllength spine films and in the posteroanterior projection, scoliotic curvatures were classified according to the Lenke classification system (16). Additionally, curve magnitudes, measured using the Cobb method, and apical and periapical curve locations were recorded. Periapical was defined as two vertebral levels above and below the apical vertebra. If the apex occurred at a disc space, the cephalad vertebral level was defined as the apical vertebra. Pedicles were subcategorized as either concave or convex according to their location relative to the curve. In the instance of a double or triple curve, end vertebrae were utilized to distinguish between concave and convex aspects of each curve. Pre-operative CT scans were reviewed, identifying all levels that underwent pedicle screw fixation. Pedicle morphology was classified using the channel classification 

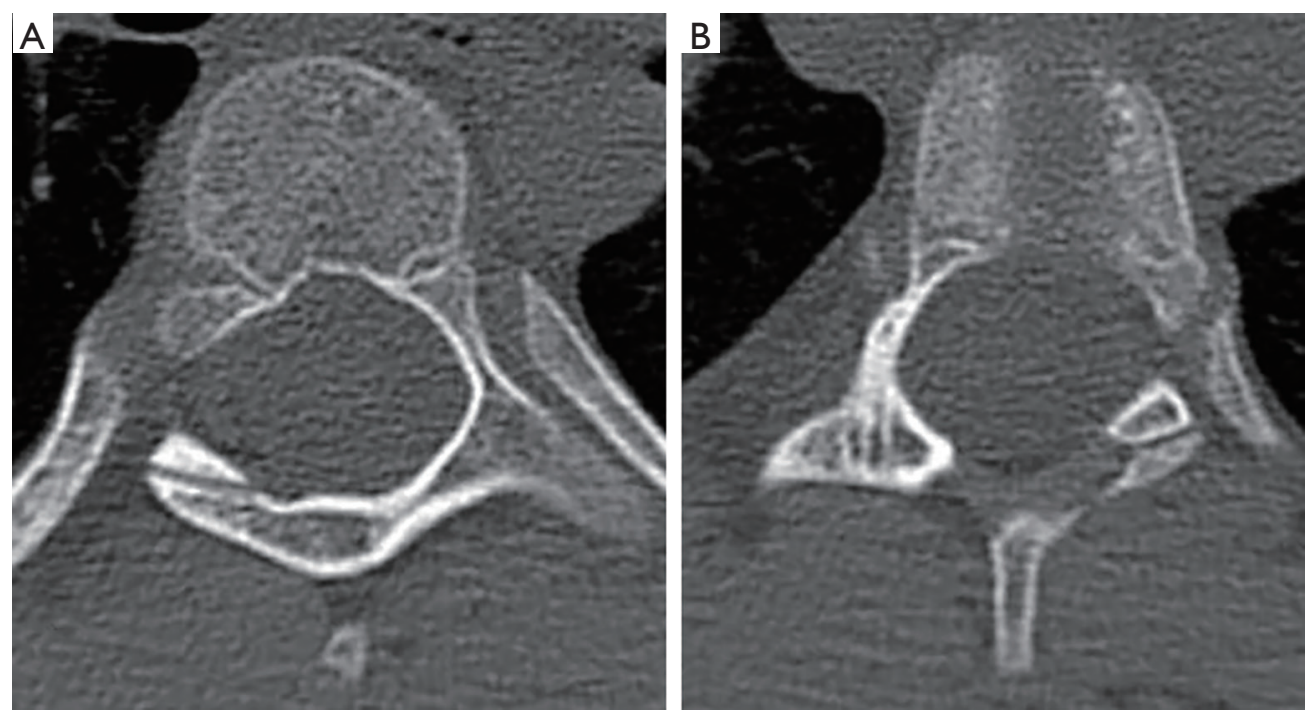

Figure 4 Axial computed tomography image of a 10+9-year-old female with a progressive 43 degree Lenke 1AL curve demonstrating type $\mathrm{C}$ (A) pedicle at the $6^{\text {th }}$ thoracic vertebral level on the convexity and a type D (B) pedicle on the concavity at the $5^{\text {th }}$ thoracic vertebral level.

system (2,17). An abnormal pedicle was defined as those with type $\mathrm{C}$ or D morphology (Figure 4). Additionally, the width of each pedicle was measured at the mid-substance.

\section{Statistical analysis}

Statistical analyses were performed using SPSS statistical software version 24 (IBM Armonk, NY). Group statistics were generated based upon recorded variables. Abnormal pedicle screw t-EMG stimulation were recorded and compared with the number of screws requiring redirection for medial malpositioning to generate sensitivity, specificity, and positive and negative predictive values. Associations between patient characteristics (age and gender), curve characteristics (curve type, magnitude, and pedicle width), abnormal pedicle morphology and positive t-EMG stimulation were examined. Pedicle measurements were further broken down based upon position relative to the concavity of the apex of the curve and compared between the concavity and convexity of the curve using Student $t$-tests. Pearson correlation coefficients were performed assessing pedicle width and pedicle t-EMG stimulation amplitudes. Logistic regression models were used to assess the effect of patient characteristics and curve characteristics on the likelihood of abnormal pedicle morphology adjusted for demographic data. All P values were two-tailed with $\mathrm{P}<0.05$ considered significant.

\section{Results}

Forty-nine patients were identified for retrospective review (11 males, 38 females, average 14.49 years) (Table 1). Curves were classified as Lenke 1 in $48.9 \%$ of patients, 3 in $24.5 \%$, 5 in $14.3 \%, 6$ in $10.2 \%$ and 2 in $2 \%$ (Table 2). A total of 844 pedicles were instrumented with pedicle screws, with an average 17.2 pedicle screws per patient. Curve magnitude was improved from a Cobb of $51.2^{\circ} \pm 8.8^{\circ}$ to $16.7^{\circ} \pm 6.9^{\circ}$ measured at the first erect post-operative for an average curve correction of $67.7 \% \pm 10.8 \%$. Overall, 28 pedicle screws were identified as abnormal, t-EMG $\leq 8 \mathrm{~mA}$. Of the abnormal pedicles, 19 were located on the concavity of the curve and $51 \%$ were periapical. All pedicles were found to have an intact medial wall following screw removal and were reinserted without redirection. t-EMG was found to have a sensitivity of $0 \%$, specificity of $100 \%$,with a negative predictive value of $29 \%$ when used in combination with robotic-assisted pedicle screw insertion. Logistic regression analysis failed to identify a patient or curve characteristic that was predictive of abnormal screw stimulation, however, stimulation amplitude was found to be positively correlated with pedicle width, with a moderate effect size (0.328).

Pedicle widths were found to vary based on the position relative to the curve concavity, with pedicles on the concavity being smaller than convex pedicles $(\mathrm{P}<0.001)$. Additionally, periapical pedicles were also smaller than surrounding pedicles $(\mathrm{P}=0.009)$. Pedicle morphology is 
Table 1 Summary of patient and curve characteristics of study participants

\begin{tabular}{|c|c|}
\hline Variable & Value \\
\hline Age (years) & $14.49 \pm 1.74$ \\
\hline \multicolumn{2}{|l|}{ Gender } \\
\hline Male & 11 \\
\hline Female & 38 \\
\hline Preoperative magnitude $( \pm S D)$ & $51.2^{\circ} \pm 8.8^{\circ}$ \\
\hline Final curve magnitude $( \pm S D)$ & $16.7^{\circ} \pm 6.9^{\circ}$ \\
\hline Instrumented pedicles & 844 \\
\hline Pedicle width $( \pm \mathrm{SD})(\mathrm{mm})$ & $3.33 \pm 0.74$ \\
\hline$<4.5$ & 654 \\
\hline$<3.5$ & 510 \\
\hline Concavity ( \pm SD) (mm) & $3.13 \pm 1.75$ \\
\hline Convexity ( \pm SD) (mm) & $3.60 \pm 1.55$ \\
\hline \multicolumn{2}{|l|}{ Pedicle morphology } \\
\hline$A$ & 667 \\
\hline $\mathrm{B}$ & 169 \\
\hline $\mathrm{C}$ & 14 \\
\hline $\mathrm{D}$ & 3 \\
\hline \multicolumn{2}{|l|}{ Abnormal tEMG stimulation } \\
\hline$\leq 8 \mathrm{~mA}$ & 24 \\
\hline Screws requiring redirection & 0 \\
\hline
\end{tabular}

tEMG, triggered screw electromyography; $\mathrm{mm}$, millimeters; $\mathrm{mA}$, milliamps; SD, standard deviation.

Table 2 Breakdown of operative curves, according to Lenke classification

\begin{tabular}{lc}
\hline Lenke classification & Number [\%] \\
\hline $1[\mathrm{~N}=24(48.9 \%)]$ & 1AR: $7[14]$ \\
& $1 \mathrm{AL}: 8[16]$ \\
& $1 \mathrm{~B}: 7[14]$ \\
& $1 \mathrm{C}: 2[4]$ \\
$2[\mathrm{~N}=1(2 \%)]$ & $2 \mathrm{AR}: 1[2]$ \\
$3[\mathrm{~N}=12(24.5 \%)]$ & $3 \mathrm{~B}: 5[10]$ \\
4 & $3 \mathrm{C}: 7[14]$ \\
$5[\mathrm{~N}=7(14.3 \%)]$ & 0 \\
$6[\mathrm{~N}=5(10.2 \%)]$ & $5 \mathrm{C}: 7[14]$ \\
\hline
\end{tabular}

summarized in Table 1. Overall, 99\% of pedicles were classified as Type A and B, 1.66\% Type C, and 0.36\% Type D. Using the operative definition of abnormal pedicles as Type C\&D morphology, logistic regression analysis was performed assessing patient and curve characteristics with only smaller pedicle width being predictive of abnormal pedicle morphology (odd's ratio $0.068, \mathrm{P}=0.005$, Table 3).

\section{Discussion}

Pedicle screw fixation is a powerful technique that allows for 3-dimensional correction of scoliotic deformities. Despite the numerous advantages that pedicle screw constructs provide, they do have a unique complication profile. Hicks et al. (18) performed a systematic review of the literature to investigate the complications associated with pedicle screw fixation, finding that screw malposition was the most common, occurring in approximately $11 \%$ of screws, but as high as $15.7 \%$ of screws when assessed with a postoperative CT for screws placed using a free-hand technique. Despite this relatively high complication rate, all-pedicle screw constructs have also been found to relate the lowest longterm complications as well as the lowest risk of revision surgery (19).

Screw malpositioning can be affected by a number of anatomic factors. Scoliotic vertebra have been found to have substantially different vertebral morphometry as evident by an asymmetric intravertebral deformity (20). Additionally the pedicular anatomy is varied between the different spinal regions with the thoracic spine having more abnormal pedicles (2). Sarwahi et al. (2) found that abnormal pedicles were more commonly located on the concavity of the curvature, at the apex and within the periapical regions. Although we found a significantly lower number of abnormal pedicles in our cohort compared with previous cohorts $(2,17)$, our data supports these prior reports with significantly smaller pedicles on the concavity of the curve and within the periapical segment.

Although rarely occurring (18), pedicle screw malpositioning can result in devastating neurologic injury. Numerous strategies have been introduced in an effort to minimize/eliminate intraoperative neurologic complications during spinal fusions for scoliosis, with sensory and motor neuromonitoring being recognized as the standard for identifying intraoperative spinal cord injury and preventing neurologic injury $(1,21,22)$. Somatosensory and motorevoked potentials (SSEP and MEP) have been found to be both sensitive (95\%) and specific $(99.8 \%)$ for identifying 
Table 3 Results of logistics regression analysis evaluating for relationships between the listed patient criteria and amplitude of t-EMG

\begin{tabular}{llcc}
\hline \multirow{2}{*}{ Variable } & \multirow{2}{*}{ Sig. } & \multicolumn{2}{c}{$95 \%$ confidence interval } \\
\cline { 3 - 4 } & & Lower & Upper \\
\hline Age & 0.362 & 0.357 & 1.457 \\
Gender & 0.391 & 0.024 & 4.334 \\
Curve type & 0.654 & 0.473 & 1.599 \\
Curve magnitude & 0.889 & 0.887 & 1.148 \\
Average pedicle width & 0.005 & 0.010 & 0.454 \\
\hline
\end{tabular}

tEMG, triggered screw electromyography.

significant sensory and motor nerve deficits during surgery (23), however, these do not necessarily identify malpositioned pedicle screws unless they impart direct spinal cord trauma.

Given the limitation of SSEP and MEP for identifying malpositioned pedicle screws, t-EMG was developed as a method to electrically stimulate the positioned pedicle screw to assess its proximity to nearby nerve roots (9). Numerous studies have investigated the reliability of t-EMG for locating malpositioned screws, identifying a specificity of 0.94 (7). Thoracic pedicles have a lower threshold for malpositioned screws in comparison to lumbar $(24,25)$. de Blas et al. (10) found that the threshold amplitude varies based upon the location of the pedicle with regard to the curve, with concave pedicles having significantly lower thresholds for malpositioned screw in comparison to convex pedicles ( $<8 v s .<11 \mathrm{~mA}$ respectively).

Although traditionally thought to indicate medial pedicle wall breaches, the data by de Blas et al. (10) suggested that decreased amplitudes were also affected by the distance of the thecal sac relative to the pedicle. Montes et al. (12) confirmed this assumption in a porcine model finding that the electrical impedance was more dependent upon the distance between the screw and the neural structures than the integrity of the medial pedicular wall. Another aspect that has yet to be investigated to the knowledge of the authors is the impact of pedicular dimensions on t-EMG amplitudes. In our AIS cohort, we found that there was a positive correlation between pedicular width and t-EMG amplitudes. Although only a moderate effect size (0.328), this data suggests that smaller pedicles could increase the incidence of false positive stimulations and thus diminish the specificity of t-EMG.

Another option to help minimize the occurrence of malpositioned screws is to incorporate navigation/guidance techniques with pedicle screw placement. Numerous studies have reported on the utility of these techniques $(6,8,11,26-28)$, although less frequently for the treatment of AIS $(6,13)$. CT navigation has been found to decrease the incidence of malpositioned screws requiring a pedicle screw revision when compared to fluoroscopic guided free hand techniques (26) with an overall $96.4 \%$ accuracy in pediatric patients (6), which has been reported to be even higher, $97.8 \%$, in patients younger than 10 years old (13). Roboticassisted pedicle screw placement is a newer technology that has also been reported to have a high accuracy, ranging from $92.8-97.9 \%(14,15,28)$. Our data aligns with these previous reports, findings of overall accuracy of $97 \%$. The combination of t-EMG was found to have a low utility in this cohort of AIS patients, finding a $0 \%$ sensitivity and $29 \%$ negative predictive value for malpositioned screws.

This data must be interpreted within the confines of its limitations. As a retrospective review, this study has inherent weaknesses in study design. Although roboticassisted pedicle screws have been found to have a high percentage of appropriately placed pedicle screws, we did not further investigate this point with a postoperative CT. Previous studies have found that clinical evaluation of pedicle screw placement underestimates malpositioned screws in comparison to post-operative CT evaluations (18). However, all screws were verified under fluoroscopy, which has been shown to be an accurate method for identification of dangerously positioned pedicle screws $(5,27,29)$. Additionally, this study only assessed for medially malpositioned screws and does not address lateral-breached screws. Additionally, this study included a small patient sample which limits the power of these results. In the setting of a larger patient size and a medially-breached screw, t-EMG may be helpful in screw identification.

t-EMG has been reported by some to have questionable reliability for thoracic segments 2-9 (3), however, this data has been refuted by other reports $(10,24,25)$. Additionally, we assessed both thoracic and lumbar pedicles using the same threshold amplitude criteria. Although previous studies have found that the thresholds vary between these spinal regions $(24,25)$, we elected to use the lowest threshold to apply to both regions in an attempt to identify the most screws at risk for malpositioning. Another factor that can affect the reliability if t-EMG is varying metallurgy of the pedicle screws (30), with titanium alloy screws from different manufactures having widespread variability in their conduction properties. However for this study, all patients 
were treated with the same top-loading polyaxial pedicle screw system. I would also include this is a single surgeon with significant experience with robot-assistance and this may change with levels of experience.

\section{Conclusions}

Robotic-assisted placement of pedicle screws is an effective and safe method in treating patients with AIS as shown by the lack of medial pedicle breach and/or malpositioned screws. t-EMG was found to a $0 \%$ sensitivity with regard to identifying medially malpositioned screws. Additionally research is needed to further characterize the role of t-EMG in robotic-assisted pedicle screw placement in AIS.

\section{Acknowledgements}

None.

\section{Footnote}

Conflicts of Interest: Dr. Murphy is a paid consultant for Depuy Spine. Dr. Devito is a paid consultant for Medicrea Spine and Mazor Surgical Technologies and receives research support from both of these entities. The authors are employees of the US government. This work was prepared as part of their official duties and, as such there is no copyright to be transferred.

Ethical Statement: This study was completed after review and approval by our Institutional Review Board.

Disclaimer: The opinions or assertions contained herein are the private views of the authors and are not to be construed as official or reflecting the views of the Department of Defense or US Government.

\section{References}

1. Vitale MG, Skaggs DL, Pace GI, et al. Best Practices in Intraoperative Neuromonitoring in Spine Deformity Surgery: Development of an Intraoperative Checklist to Optimize Response. Spine Deform 2014;2:333-9.

2. Sarwahi V, Sugarman EP, Wollowick AL, et al. Prevalence, Distribution, and Surgical Relevance of Abnormal Pedicles in Spines with Adolescent Idiopathic Scoliosis vs. No Deformity: A CT-Based Study. J Bone Joint Surg Am 2014;96:e92.
3. Samdani AF, Tantorski M, Cahill PJ, et al. Triggered electromyography for placement of thoracic pedicle screws: is it reliable? Eur Spine J 2011;20:869-74.

4. Calancie B, Donohue ML, Moquin RR. Neuromonitoring with pulse-train stimulation for implantation of thoracic pedicle screws: a blinded and randomized clinical study. Part 2. The role of feedback. J Neurosurg Spine 2014;20:692-704.

5. Choma TJ, Denis F, Lonstein JE, et al. Stepwise methodology for plain radiographic assessment of pedicle screw placement: a comparison with computed tomography. J Spinal Disord Tech 2006;19:547-53.

6. Larson AN, Santos ER, Polly DW Jr, et al. Pediatric pedicle screw placement using intraoperative computed tomography and 3-dimensional image-guided navigation. Spine 2012;37:E188-94.

7. Mikula AL, Williams SK, Anderson PA. The use of intraoperative triggered electromyography to detect misplaced pedicle screws: a systematic review and metaanalysis. J Neurosurg Spine 2016;24:624-38.

8. Schatlo B, Molliqaj G, Cuvinciuc V, et al. Safety and accuracy of robot-assisted versus fluoroscopy-guided pedicle screw insertion for degenerative diseases of the lumbar spine: a matched cohort comparison. J Neurosurg Spine 2014;20:636-43.

9. Stecker MM. A review of intraoperative monitoring for spinal surgery. Surg Neurol Int 2012;3:S174-87.

10. de Blas G, Barrios C, Regidor I, Montes E, et al. Safe pedicle screw placement in thoracic scoliotic curves using t-EMG: stimulation threshold variability at concavity and convexity in apex segments. Spine 2012;37:E387-95.

11. Kassis SZ, Abukwedar LK, Msaddi AK, et al. Combining pedicle screw stimulation with spinal navigation, a protocol to maximize the safety of neural elements and minimize radiation exposure in thoracolumbar spine instrumentation. Eur Spine J 2016;25:1724-8.

12. Montes E, De Blas G, Regidor I, et al. Electromyographic thresholds after thoracic screw stimulation depend on the distance of the screw from the spinal cord and not on pedicle cortex integrity. Spine J 2012;12:127-32.

13. Luo TD, Polly DW Jr, Ledonio CG, et al. Accuracy of Pedicle Screw Placement in Children 10 Years or Younger Using Navigation and Intraoperative CT. Clin Spine Surg 2016;29:E135-8.

14. van Dijk JD, van den Ende RP, Stramigioli S, et al. Clinical pedicle screw accuracy and deviation from planning in robot-guided spine surgery: robot-guided pedicle screw accuracy. Spine 2015;40:E986-91. 
15. Macke JJ, Woo R, Varich L. Accuracy of robot-assisted pedicle screw placement for adolescent idiopathic scoliosis in the pediatric population. J Robot Surg 2016;10:145-50.

16. Lenke LG, Betz RR, Harms J, et al. Adolescent idiopathic scoliosis: a new classification to determine extent of spinal arthrodesis. J Bone Joint Surg Am 2001;83-A:1169-81.

17. Watanabe K, Lenke LG, Matsumoto M, et al. A novel pedicle channel classification describing osseous anatomy: how many thoracic scoliotic pedicles have cancellous channels? Spine 2010;35:1836-42.

18. Hicks JM, Singla A, Shen FH, et al. Complications of pedicle screw fixation in scoliosis surgery: a systematic review. Spine 2010;35:E465-70.

19. Lykissas MG, Jain VV, Nathan ST, et al. Mid- to longterm outcomes in adolescent idiopathic scoliosis after instrumented posterior spinal fusion: a meta-analysis. Spine 2013;38:E113-9.

20. Liljenqvist UR, Link TM, Halm HF. Morphometric analysis of thoracic and lumbar vertebrae in idiopathic scoliosis. Spine 2000;25:1247-53.

21. Pastorelli F, Di Silvestre M, Plasmati R, et al. The prevention of neural complications in the surgical treatment of scoliosis: the role of the neurophysiological intraoperative monitoring. Eur Spine J 2011;20 Suppl 1:S105-14.

22. Schwartz DM, Auerbach JD, Dormans JP, et al. Neurophysiological detection of impending spinal cord injury during scoliosis surgery. J Bone Joint Surg Am 2007;89:2440-9.

Cite this article as: Shaw KA, Murphy JS, Devito DP. Accuracy of robot-assisted pedicle screw insertion in adolescent idiopathic scoliosis: is triggered electromyographic pedicle screw stimulation necessary? J Spine Surg 2018;4(2):187-194. doi: $10.21037 /$ jss.2018.04.01
23. Thirumala PD, Bodily L, Tint D, et al. Somatosensoryevoked potential monitoring during instrumented scoliosis corrective procedures: validity revisited. Spine J 2014;14:1572-80.

24. Raynor BL, Lenke LG, Kim Y, et al. Can triggered electromyograph thresholds predict safe thoracic pedicle screw placement? Spine 2002;27:2030-5..

25. Shi YB, Binette M, Martin WH, et al. Electrical stimulation for intraoperative evaluation of thoracic pedicle screw placement. Spine 2003;28:595-601.

26. Hodges SD, Eck JC, Newton D. Analysis of CTbased navigation system for pedicle screw placement. Orthopedics 2012;35:e1221-4.

27. Kim YJ, Lenke LG, Cheh G, et al. Evaluation of pedicle screw placement in the deformed spine using intraoperative plain radiographs: a comparison with computerized tomography. Spine 2005;30:2084-8.

28. Kuo KL, Su YF, Wu CH, et al. Assessing the Intraoperative Accuracy of Pedicle Screw Placement by Using a Bone-Mounted Miniature Robot System through Secondary Registration. PloS One 2016;11:e0153235.

29. Piazzolla A, Montemurro V, Bizzoca D, et al. Accuracy of plain radiographs to identify malpositioned free hand pedicle screw in the deformed spine. J Neurosurg Sci 2016. [Epub ahead of print].

30. Donohue ML, Swaminathan V, Gilbert JL, et al. Intraoperative neuromonitoring: can the results of direct stimulation of titanium-alloy pedicle screws in the thoracic spine be trusted? J Clin Neurophysiol 2012;29:502-8. 